\title{
Symptomatic pseudarthrosis in ochronotic spine: case report
}

\author{
Abolfazl Rahimizadeh, MD, ${ }^{1}$ Housain Soufiani, MD, ${ }^{1}$ Valliolah Hassani, MD, ${ }^{1}$ and \\ Ava Rahimizadeh, MS²
}

\begin{abstract}
${ }^{1}$ Pars Advanced and Minimally Invasive Manners Research Center, Pars Hospital, Iran University of Medical Sciences, Tehran, Iran; and ${ }^{2}$ Birmingham Medical School, Birmingham, United Kingdom
\end{abstract}

\begin{abstract}
In this study the authors report the first example of spinal pseudarthrosis in a patient with ochronosis, and they describe the application of posterior-only $360^{\circ}$ surgery as an alternative approach to combined anterior-posterior surgery in the management of pseudarthrosis of an ankylosed spine, regardless of its etiology.

Spinal involvement in ochronosis produces loss of flexibility and ankylosis of thoracic and lumbar segments. Pseudarthrosis is a serious complication of the diseases that present with ankylosis of the spine. However, its occurrence in ochronotic spine has not been reported previously.

Evaluation of progressive paraparesis in a 68-year-old man with ochronosis revealed pseudarthrosis at the T11-12 level. Circumferential dural sac decompression, debridement of the disc space, interbody fusion, and screw-rod fixation were all done via a posterior-only approach. Postoperatively the patient exhibited a marked recovery in terms of pain and neurological status. At the 3-month follow-up, he was able to walk independently.

Ochronosis should be included in the etiology of pseudarthrosis. With aggravation of back pain and the appearance of neurological deficits in an already stable patient with any ankylosing disease, pseudarthrosis should be suspected. Furthermore, single-stage, $360^{\circ}$, posterior-only surgery may obviate the need for single-stage or staged anterior-posterior surgical intervention in patients with pseudarthrosis of the thoracic and lumbar spine.
\end{abstract}

https://thejns.org/doi/abs/10.3171/2016.5.SPINE15671

KEY WORDS alkaptonuria; ankylosing spondylitis; ochronosis; pseudarthrosis; deformity

$\mathrm{D}$ EPOSITION of ochronotic material in intervertebral discs of patients with alkaptonuria results in disc degeneration and marginal intervertebral bridge formation, which eventually causes spinal stiffness and ultimately ankylosis, quite similar to seronegative spondyloarthropathies. $1,4,5,10,19,40,43,54,59$

Pseudarthrosis, designated as Andersson disease, is a well-known complication of a long-standing ankylosing spondylitis (AS), ${ }^{7,11,50,57,67,68,71}$ Actually, pseudarthrosis is a mobile nonunion state that usually occurs at discovertebral elements, mostly in the dorsolumbar region., $71,50,57,67,68,71$ The clinical manifestation of these lesions is disabling pain and progressive neurological deficit, with a decrease of the preexisting stooping deformity of AS. However, pseudarthrosis is not confined to AS, and it might be seen in the advanced course of any other disease manifested with pseudoblocked vertebrae (in other words, segments that are pseudofused due to the disease). . $^{8,27,30,41,45,64}$

In this study, a 68-year-old man with back pain and progressive paraparesis is presented. With demonstration of discolored sclera and ears as well as dark urine, a diagnosis of ochronosis was made. Imaging confirmed ochronotic spine as well as pseudarthrosis at the T11-12 region. One-stage posterior $360^{\circ}$ fusion instrumentation resulted in a remarkable neural recovery. In a careful review of the literature, we encountered no case of an ochronotic patient being affected with pseudarthrosis.

\section{Case Report}

History

When this 68-year-old man was referred to our clinic,

ABBREVIATIONS AS = ankylosing spondylitis; DISH = diffuse idiopathic skeletal hyperostosis; $\mathrm{HGA}=$ homogentisic acid oxidase; IBD = inflammatory bowel disease; SP = spinal pseudarthrosis.

SUBMITTED June 13, 2015. ACCEPTED May 13, 2016.

INCLUDE WHEN CITING Published online October 14, 2016; DOI: 10.3171/2016.5.SPINE15671. 
he used a wheelchair because of severe back pain and progressive weakness of his lower extremities, and he suffered from incontinence of 6 months' duration. His weakness was such that he was unable to walk, and a Foley catheter had been used for his incontinence. He had a long history of mild back pain, with progressive restriction of the movements of the thoracic and lumbar spine in the last 20 years.

\section{Examination}

Careful clinical examination disclosed that his ear and sclera had blue-black pigmentation (Fig. 1), and the urine inside the urine collection bag was black (Fig. 2). Therefore, a clinical diagnosis of alkaptonuria was made, which was confirmed later with positive findings for homogentisic acid oxidase (HGA) in urine.

Neurological examination revealed spastic paraparesis with hyperactive reflexes and upgoing toes. Sensory level at approximately T-12 was noted. Notably, we discovered that several members of his immediate family suffer from the disorder, according to the history.

A radiological survey of the thoracolumbar spine revealed characteristic features of ochronosis, including pseudoblocked vertebrae and intervertebral disc space narrowing, with their marked calcification or wafer-like appearance. Furthermore, the T11-12 intervertebral disc space was widely opened, with sclerotic margins (Fig. 3).

The T1-weighted sagittal MR images showed hypointensity at the defect; hypointensity was extended posteriorly to the corresponding interlaminar soft ligamentous tissues. The T2-weighted sagittal images demonstrated a hyperintensity at the defect anteriorly, whereas the posterior side was hypointense (Fig. 4). After diagnosis of pseudarthrosis at the T11-12 region, surgical intervention was proposed, which was accepted.

\section{Operation}

After induction of general anesthesia and with the patient placed prone, a midline incision was made at the thoracolumbar region from T-9 to L-2. After stripping off the muscles from the laminae, a long-segment pedicle screw construct from T-9 to L-2 seemed mandatory because of coexisting osteopenia. With a rod on the right side, we used partial laminectomy at the T11-12 interspace to release the dural sac posteriorly from the pressure caused by black-pigmented fibrous tissues and the destroyed ligamentum flavum.

Subsequently, the corresponding disc at the T11-12 level was targeted through the left transfacet pedicle-sparing corridor. Removal of the T-12 transverse process, disarticulation of the left 12 th costovertebral joint, and removal of the medial $2 \mathrm{~cm}$ of the corresponding rib head allowed more room to access the T11-12 intervertebral disc space as well as the lateral surfaces of the corresponding vertebral bodies. Via this extended exposure, the disc space was widely opened and the remnants of sequestered disc and fibrous tissues, both stained with black pigments, could be removed. The anterior aspect of the dural sac was decompressed with a reverse-angle curette. This resulted in pulsation of the dura mater, indicating adequate decompression. With curettage of the sclerotic endplates,

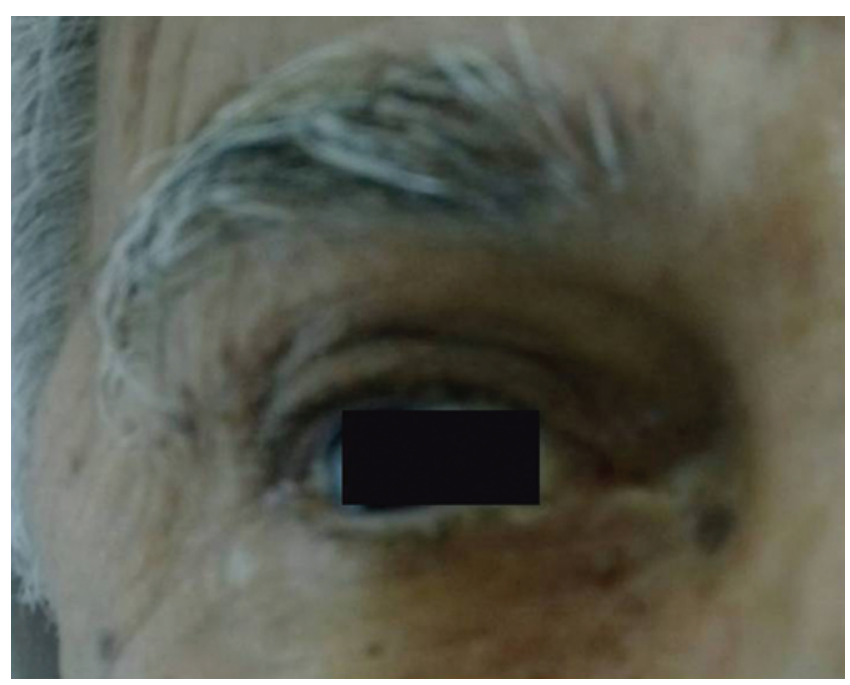

FIG. 1. Photograph showing discoloration of the sclera. Figure is available in color online only.

a proper bed for autogenous bone chips and a cage was provided.

Later, after applying distraction on the T11-12 pedicle screws on the left side, the space was sufficiently opened to permit coverage of the floor of the gap with small allograft bone chips mixed with bone marrow aspirate, as well as placement of an appropriately sized banana-shaped cage filled with bone graft. Subsequently the left rod was assembled and the nuts were tightened, with application of compression on the screws to secure the cage (Fig. 5).

\section{Postoperative Course}

Postoperatively the patient showed marked recovery; at discharge on the 10th postoperative day, he was able to walk with a walker. Biopsy sampling of the defect disclosed fibrous tissue with foci of pigmentation (Fig. 6). At 3-months follow-up, results of his neurological examination were quite satisfactory and he was able to walk independently. The control lateral thoracolumbar radiograph showed good stabilization and alignment, with proper location of the cage (Fig. 7). The patient's status was followed via periodic phone calls. Unfortunately, we heard that he had died of a cardiac arrest approximately 10 months after surgery. Autopsy was not permitted.

\section{Discussion}

Alkaptonuria is a rare hereditary autosomal recessive disorder characterized by the absence of HGA. ${ }^{1}$ Renal clearance of HGA is maintained very effectively in youth. This efficiency is decreased with aging, which eventually leads to the deposition of HGA throughout the body, particularly in the fibrous tissues and cartilages. . $^{4,19,34,38,40,59}$ With deposition of this material, the color of cutaneous tissues turns to blue-black as progressive arthropathy occurs, and urine darkens with exposure to air. ${ }^{25,46,57,59} \mathrm{At}$ this stage, which is usually during the 4th decade of life, the term ochronosis is used. . $^{4,5,19,34,38,40}$

This is quite different from AS, wherein the symptoms start in late adolescence and early adulthood, and consist 


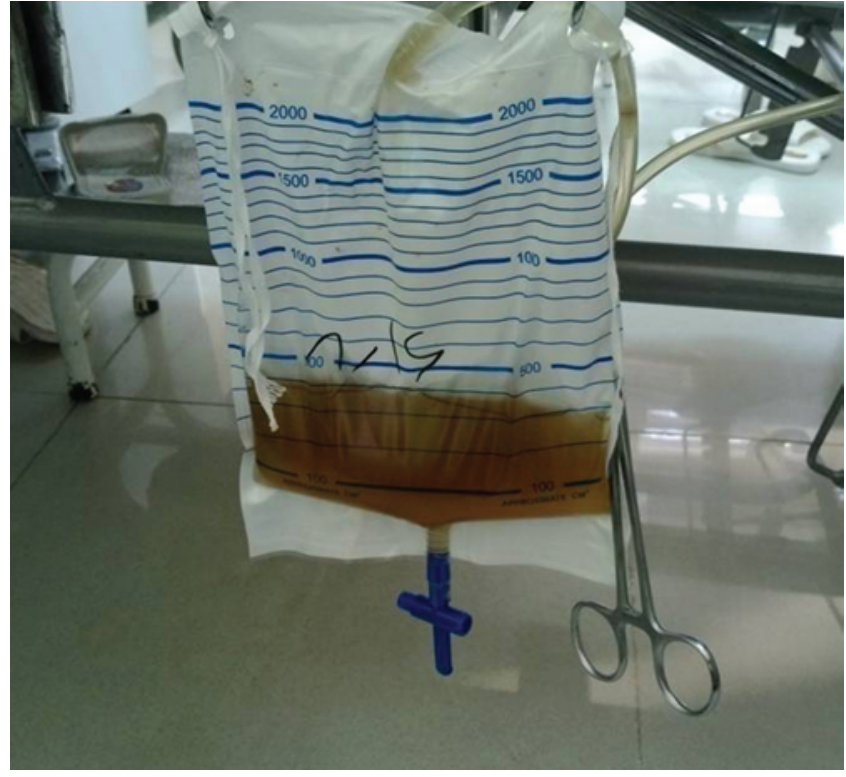

FIG. 2. Dark brown urine is demonstrated in a photograph of the urine bag. Figure is available in color online only.

of inflammatory spinal pain and stiffness, sacroiliac pain, and decreasing range of spinal motion entailing characteristic postural abnormalities., ${ }^{7,64}$ It also differs from diffuse idiopathic skeletal hyperostosis (DISH), because stiffness occurs late, usually in the elderly. ${ }^{27,30}$ In patients with psoriasis and inflammatory bowel disease (IBD), ankylosis usually begins in early middle age. ${ }^{8,41,64}$ Surprisingly, all ankylosing states, including ochronotic spondyloarthropathy, are more frequent in males.

Musculoskeletal involvement by alkaptonuria is likely to be noted first in the spine. ${ }^{4,5,19,34,38,40}$ Spinal involvement, which in particular occurs at the lumbar and thoracic regions, is started with disc degeneration resulting from the redeposit of HGA in the disc space.

Similar to ochronosis, the thoracolumbar region is the most frequently affected site in AS. Even in patients with psoriasis and IBD, spondylitis as well as DISH occur despite the fact that ankylosis starts in the cervicothoracic region, but pseudarthrosis in the majority of the reported cases has been located in the thoracolumbar region. $8,27,30,41,45,64$

Generally, the clinical picture of ochronosis with regard to the vertebral column is consistent with the pathology retrospectively manifested with back pain and progressive restriction of the spinal movements. ${ }^{4,5,19,34,38,40,59}$ In rare instances, the disc affected by deposition of the pigments becomes degenerated and might protrude or extrude posteriorly, with a clinical picture of disc herniation. ${ }^{10,20,23,24,28,35,47,62}$ The scenario of disc herniation usually occurs in the lumbar region and, since Feild et al.'s report in 1963, 9 more cases have been published so far. ${ }^{20,23,24,28 \text {, }}$ $35,44,60$

Herniation of an ochronotic disc at the thoracic spine causing myelopathy is extremely rare, and has been reported on only 1 occasion..$^{63}$ It should be noted that herniated disc has not been seen in other ankylosing states. However, in the majority of patients with ochronosis, degeneration

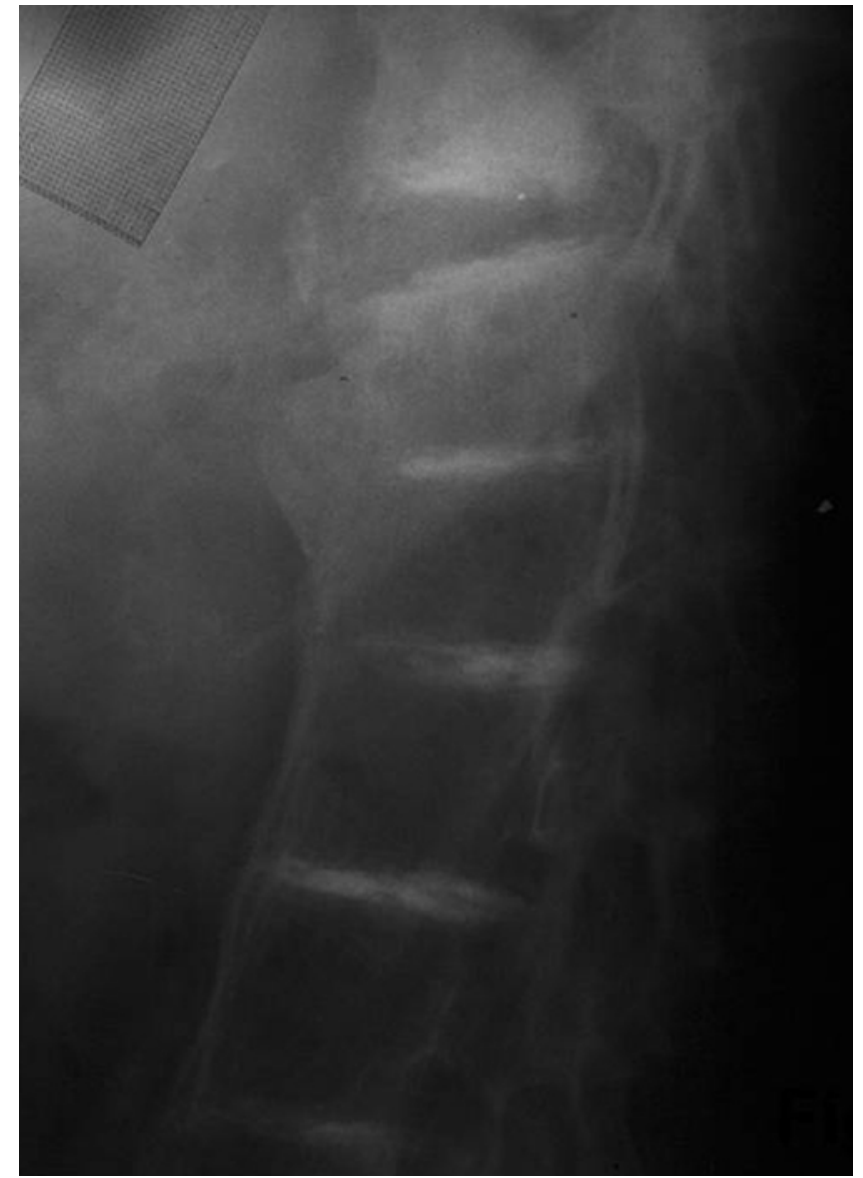

FIG. 3. Plain radiograph, lateral view, demonstrating calcification of narrow disc spaces. Note a wide gap at T11-12 levels and marked sclerosis at the margins.

of the discs caused by deposition of the pigments occurs very slowly, resulting in narrowness of the disc space and its calcification. ${ }^{25}$ Surprisingly, narrow disc space is only seen in ochronosis and is absent in other ankylosing disorders. Syndesmophyte formation secondary to progressive disc degeneration, combined with ossification of the anterior longitudinal, interspinous, and flaval ligaments, results in stiffness of the vertebral column. Shortness of the anterior column, stooping, and kyphosis, which are the ultimate result in other ankylosing disorders, are less severe in ochronosis. . $^{6,32,39,51,58}$ In such circumstances, the clinical picture with regard to the vertebral column is consistent with the pathology manifested with back pain, accompanied with progressive restriction of the spinal movements. Rarely, multilevel degeneration and protrusion may cause spondylosis, resulting in neurogenic claudication in the lumbar region. However, multilevel thoracic spondylosis entailing myelopathy is an extremely rare event in ochronosis. ${ }^{3,47,48}$ Nonetheless, the overall alignment of the spine in long-standing ochronosis might be mild kyphosis, which is not usually confused with AS. ${ }^{6,31,32,34,51}$

The most frequent extraspinal joint involvement in ochronosis is symmetrical sacroiliac pain. Peripheral joint arthritis develops in the late stage and generally affects large joints such as the hip and knee. By the age of 55 

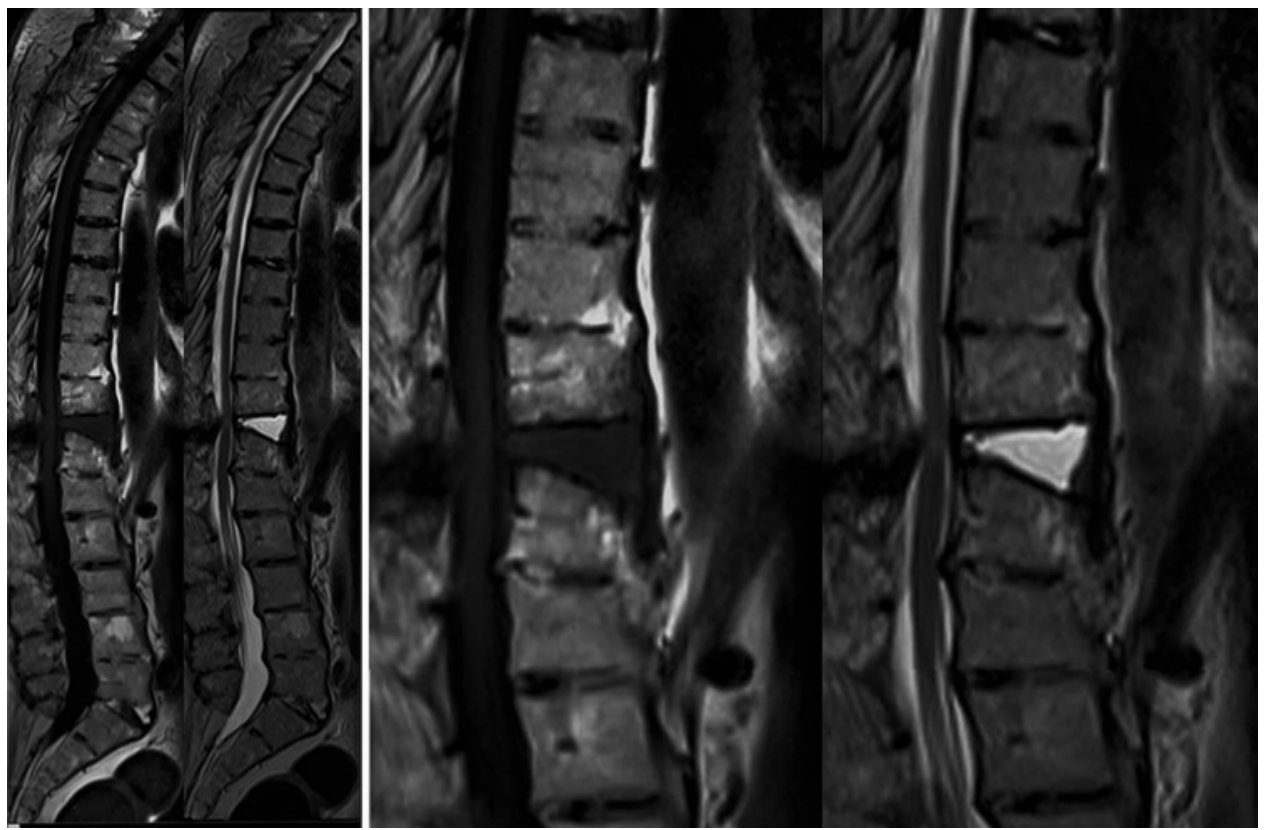

FIG. 4. Left: Thoracolumbar MR images. A T1-weighted sagittal image (left panel) showing a hypointense lesion at the site of pseudarthrosis; note that the lesion is extended to the posterior column. A T2-weighted sagittal MR image (right panel) in which pseudarthrosis is demonstrated as a hyperintense lesion that is extended to the posterior elements as a hypointense defect. In both images markedly narrow discs are seen. Right: In a closer view, all MRI changes are more clearly demonstrated.

years, $50 \%$ of these patients have a history of hip or knee arthroplasty. Tendonitis related to pigment accumulation in collagen-rich tendons of the large joints results in limitation of the active and passive movements of these joints. Tendon involvement is not the cardinal feature of AS. Nonetheless, in patients with psoriasis and IBD, sacroiliitis is asymmetrical and only small joints are affected. . $^{41,64}$ In DISH, sacroiliac pain might be seen but peripheral joint involvement due to this disease is not demonstrated, and if patients are affected, it is age related..$^{27,30}$

The characteristic radiological features of the spine are as follows. The disc spaces are markedly narrowed and the discs are heavily calcified in the periphery, with a

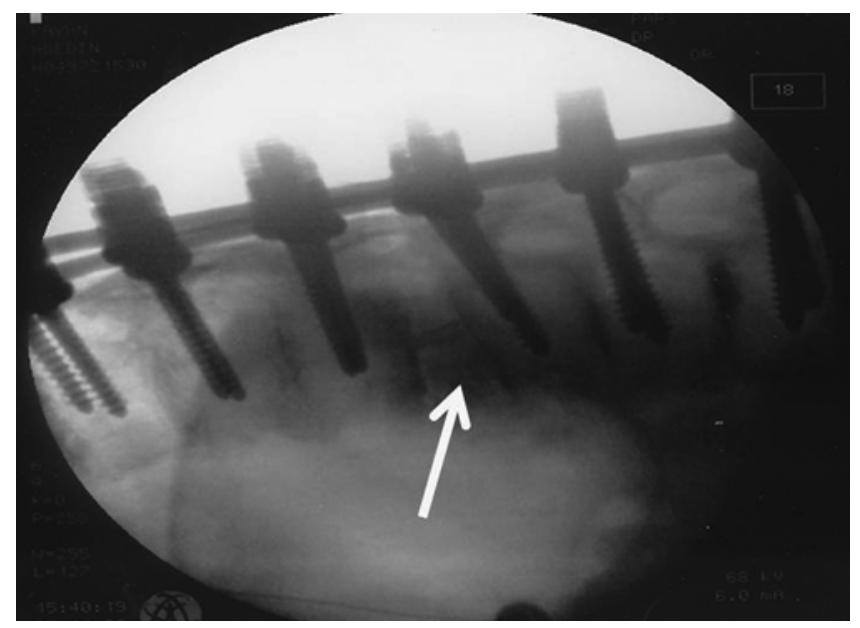

FIG. 5. Intraoperative fluoroscopic view showing that the floor of the gap is packed with bone chips, and a cage has been placed at the middle of the gap (arrow). Note long-segment instrumentation. wafer-like appearance. ${ }^{6,32,34,39,40,51}$ The presence of multiple so-called vacuum discs is another striking feature of spinal ochronosis. ${ }^{17}$ However, with progression of the disease, marginal sclerosis of the vertebral bodies accentuated by osteopenia and reactive marginal osteophytosis results in bony bridges. Occurrence of these bridges at multiple levels is known as pseudoblocked vertebrae. . $^{6,32,39,40,51,58}$

Some radiographic similarities, such as the presence of squared, osteopenic vertebral bodies and bridging syndesmophytes, are frequently seen in other diseases that manifest with ankylosed spine. ${ }^{9,16,34,55}$ This can easily result in confusion in establishing the correct diagnosis, in particular in the disease's early stages. However, there are distinctive features that play an important role in the differential diagnosis of ochronosis from seronegative spondyloarthropathies such as AS, psoriasis, and IBD, as well as other spondylopathies such as DISH. Actually, in these diseases the height of disc spaces remains normal-in contrast to ochronosis, in which intervertebral discs are markedly narrowed and calcified. 5,9,16,26,34,55 The involvement of apophyseal joints, which starts from inflammation and continues to ultimate fusion, favors a diagnosis of seronegative spondyloarthropathies rather than DISH and ochronosis., ${ }^{96,34,55}$ Furthermore, a bamboo-like appearance, which is due to anular ossification in AS, is not demonstrated in ochronosis.

Moreover, complete ankylosis of sacroiliac joints, consistent with Grade IV sacroiliitis, is one of the striking features of AS. In ochronosis, symmetrically affected sacroiliac joint demonstrated as sclerosis is frequent, but the joints are never fused. In these diseases, sacroiliac joints are affected symmetrically because asymmetrical sclerosis of these joints and fusion of the joints never occurs. ${ }^{32-34}$ Enthesopathic changes manifested as whisker-like new 


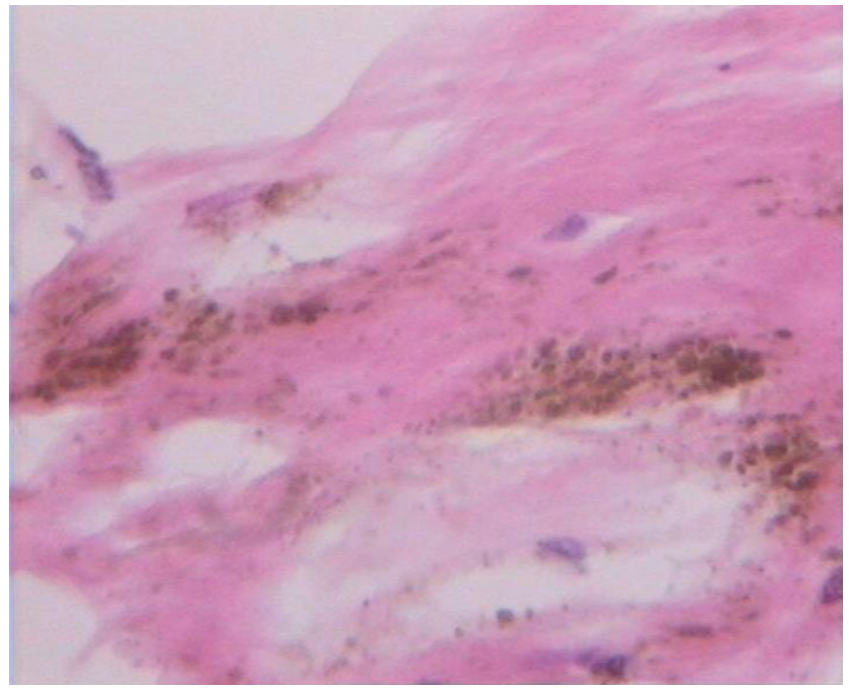

FIG. 6. Photomicrograph of a pathological specimen, showing foci of pigmentation in a fibrous bed. $H \& E$, original magnification $\times 400$. Figure is available in color online only.

bone formation at the ischial tuberosities and sclerosis at both lesser trochanters are only seen in AS and are not demonstrated in other ankylosing disorders.

Reconstructed CT images can clearly show the characteristic features such as abundant marginal osteophyte formation, as well as narrowed disc spaces and their calcification. Multiple vacuum phenomena inside the disc, which is another feature of ochronosis, might be demonstrated on CT scans. ${ }^{6,31}$

On MR images, in the early stages the intensity of the affected disc usually does not differ from those being degenerated without ochronosis. In this period the involved discs are uniformly isointense on T1-weighted images, suggesting widespread degeneration of the vertebral column. ${ }^{6,15,29,31,63,65}$ Late in the disease, the striking feature is the narrowing of the disc spaces, which is accompanied with hypointensity of the disc material on T2-weighted images due to calcification. However, if the vacuum phenomenon is present in the disc space, it might be demonstrated as hyperintensity. ${ }^{17}$

There is a low tendency for patients with ochronotic spondylosis to have positive findings on the HLAb27 serum assay. However, this does not mean there is an association of this disease with AS, in particular if it is compared with the frequency of $7 \%-8 \%$ found in the Caucasian population..$^{916,26,55,63}$ In very rare instances in which an individual with ochronosis has some features of AS and positive HLAb27 findings, the distinct clinical aspect and pathognomonic radiological features of ochronosis are the only clues for accurate diagnosis., ${ }^{5,26,55}$

\section{Overview of Pseudarthrosis}

One of the most serious complications of an ankylosed spine is the destructive discovertebral lesion designated as pseudarthrosis. $2,7,11,18,21,22,36,50,52,53,66,67$

\section{History}

Pseudarthrosis is a well-known complication of anky-
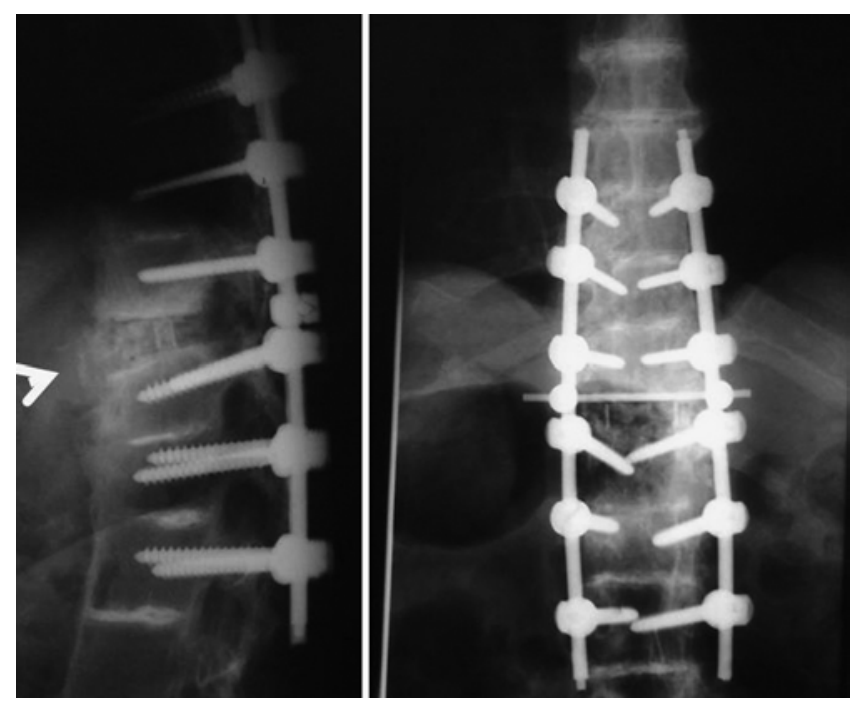

FIG. 7. Postoperative lateral plain radiograph obtained at 3-month follow-up demonstrating the bone chips and cage in the gap (arrow), and long-segment screw-rod fixation.

losing diseases of the spine, which usually occurs as a localized discovertebral destructive lesion. . $7,711,18,21,22,36,50,52,53,66,67^{2}$ This pathology was first described by Andersson in 1937 in AS. However, sterile spondylodiscitis, Andersson lesion, and destructive discovertebral stress fracture are the terms that have been used to describe similar conditions. Pseudarthrosis is mostly seen in AS, but it is not confined to this disease and can occur in other ankylosing disorders such as DISH, psoriasis, and IBD.

\section{Incidence}

Although the estimated prevalence of this complication in AS ranges from $1 \%$ to $16 \%, 2,7,18,22,36,56$ its occurrence in DISH, psoriasis, and IBD seems to be extremely low. $8,27,30,41,45,60,64$ Pseudarthrosis in DISH was first reported by Quagliano et al. in 1994; they reported 2 cases of spontaneous pseudarthrosis with DISH in the thoracolumbar region, and since then only 4 more cases have been published so far. $27,30,45,61$

Pseudarthrosis in patients with psoriasis was first reported by Schilling et al. in 1990, and it was reported in patients with IBD by Calin and Robertson in 1991. Since then, only a few cases of pseudarthrosis due to these 2 diseases have been published so far. ${ }^{8,41,45,61}$ However, to the best of our knowledge, not a single case of pseudarthrosis as a sequela of ochronosis has been published previously. ${ }^{69}$

\section{Pathogenesis}

Two different etiologies have been postulated for development of pseudarthrosis in ankylosing states., ${ }^{722,70} \mathrm{~A}$ theory that has become more widely accepted combines the inflammatory process with additional mechanical effects such as repeated stress and minor traumas. According to this theory, the forces that occur as a result of daily activities are not equally distributed over all discovertebral joints in an ankylosed spine. This means that there are areas that are skipped and segments that have less extensive bony bridging. ${ }^{7,22,70}$ These local areas of weakness 
are more exposed to daily stresses, which permit an excessive degree of mobility associated with inflammation. The consequence of increased gravitational stress concentration and repetitive minor traumas at these inflamed sites is to provoke disruption. Subsequent replacement and repair of the defect by fibrous tissues results in local nonunion or pseudarthrosis. Actually, in up to $70 \%$ of cases, pseudarthrosis occurs spontaneously, which can be explained by the above-mentioned theory. ${ }^{7}$ However, events such as trivial falls or minor blunt traumas might occur long enough before the development of symptoms in the remaining $30 \%$ to support the trauma theory. ${ }^{22}$

Regardless of the cause of pseudarthrosis, discovertebral destruction and its subsequent replacement with fibrous tissue starts at the intervertebral disc and extends posteriorly to the posterior ligamentous structures and the apophyseal joints., ${ }^{2,12}$ Spinal pseudarthrosis (SP) generally develops over many months or years after the corresponding vertebral column has been affected by an ankylosing disease. An SP characteristically involves a single vertebral level, and involvement of 2 or more levels is seen occasionally. The thoracolumbar segments, being subjected to the highest stress concentration in an ankylosed spine, are the most common site of pseudarthrosis. ${ }^{7,22,37,52,53,67}$ In fact, thoracolumbar segments are the most common site of pseudarthrosis in all ankylosing states. , $27,30,41,61$

Despite the fact that in patients with psoriasis, IBD, and DISH, ankylosis starts from the cervicothoracic region and extends gradually toward the thoracolumbar region only in later stages, the cervical segments remain the least susceptible location for development of SP. ${ }^{27,64}$

\section{Classification}

In 1972, Cawley et al. classified SP found in patients with AS into 3 types according to the lesion's localization: Type I (central), Type II (peripheral), or Type III (extensive). The first 2 types occur earlier in the course of the disease, and the extensive type (Type III) happens late. Another synonym for a Type I or central lesion is discovertebral, and for Type II or peripheral lesions another term is the endplate-destructive type. In the extensive type or Type III, coexistence of a discovertebral lesion and extensive destruction of the endplates of the corresponding vertebrae are demonstrated. Regardless of the type, destruction is usually extended to posterior elements. ${ }^{11}$ Actually, the presence of a defect in posterior elements is an essential component in the development of an Andersson lesion.

\section{Age and Sex}

Surprisingly, pseudarthrosis seems to be more common in male than in female patients in all ankylosing disorders. Occurrence of pseudarthrosis in AS varies from early adulthood to middle age. However, pseudarthrosis in patients with psoriasis and IBD has been seen in the middleaged group, whereas in patients with DISH it occurs only in the elderly. ${ }^{8,27,30,41,45,61,64}$

\section{Clinical Manifestation}

The appearance of sharp and persistent back pain in patients who are already suffering from stiff back and kyphosis is the cardinal feature of pseudarthrosis in all anky- losing states. Severe back pain originates from the site of instability, with local tenderness in percussion at the level of pseudarthrosis. Pain usually diminishes with rest and increases with motion.,22,66,67 If pseudarthrosis occurs at the apex of kyphosis, this deformity might be corrected, at least partially.

Both pain and neurological symptoms are secondary to the compressive effect of abnormal fibroosseous tissue proliferation, destroyed disc, endplates, and yellow ligaments on the dural sac circumferentially, , $7,22,66,67$ In up to $20 \%$ of patients pseudarthrosis might remain asymptomatic, particularly in the midthoracic region. ${ }^{22,42,56}$ The explanation is that the process of destruction ceases and pseudarthrosis becomes inactive. ${ }^{42}$

\section{Pathological Findings}

These lesions have a variable histological appearance. Usually the intervertebral disc space is replaced by fibrous tissue. Fragments of necrotic bone and cartilage are often present inside the specimen. Mild inflammatory changes with infiltration of plasma cells, lymphocytes, and macrophages might be found in vertebral borders. ${ }^{2,22}$ This abnormal fibrous tissue has tight adherence to the dura mater both anterior and posterior to the theca. In ochronosis, black pigmentation is seen in the fibrous tissue.

\section{Imaging Studies}

Plain Radiographs. In both symptomatic and asymptomatic subjects, disruption of syndesmophyte, osteolysis of the endplates, and widened intervertebral disc space surrounded by reactive sclerotic changes are seen at the site of pseudarthrosis. ${ }^{12,18,21,36,49,50,53,67}$ Osteolytic destruction is shown as a translucent line that starts from the disc and extends to the posterior element. The lesion might partially correct the preexisting round or angular kyphosis. ${ }^{12,18,49,53}$ These features of pseudarthrosis are not confined to AS. A similar picture is seen in other ankylosing states, as was demonstrated in our patient with ochronosis. . $^{87,30,41,45,61,64}$

Although the diagnosis of pseudarthrosis or Andersson lesion might be established on conventional radiography, the advent of new imaging techniques such as MRI and spiral CT offers the possibility of new approaches to defining the radiological features and providing additional necessary information about symptomatic and asymptomatic pseudarthrosis.

Computed Tomography. Computed tomography is superior to conventional radiography in determining the extent of pseudarthrosis, in particular involvement of the posterior elements, which will probably not be visible on plain radiographs., ${ }^{72,36,53,67}$ In reconstructed sagittal CT scans, pseudarthrosis is depicted as a wide bony gap in the spinal column extending from anterior to posterior and demonstrated as irregular vertebral or discovertebral osteolysis with reactive sclerosis. Variable osteophyte formation toward the neural canal, nonfusion of the facet joints, and vacuum phenomena are other features in reformatted images. However, differentiation between symptomatic and asymptomatic cases is not possible with the application of this modality. ${ }^{7,12,36}$

Magnetic Resonance Imaging. This is considered to be 
the best modality for detection of pseudarthrosis; it is the one with the highest sensitivity. $7,36,38,52,66$ In symptomatic cases, the affected intervertebral disc space is demonstrated as an irregular lesion with decreased signal intensity on T1-weighted MRI. The corresponding vertebral bodies are hypointense at the borders surrounding the affected region. These borders show rim enhancement on Gd-enhanced T1-weighted MRI, and the vertebral body signals are slightly increased.

This hypointensity on T1-weighted images turns to hyperintensity on T2-weighted images. ${ }^{7,36,38,52,66}$ Infrequently, pseudarthrosis may be exhibited as a heterogeneous lesion, depending on the consistency of the necrotic tissues. ${ }^{21,38,52,53,67}$ The vertebral body signal adjacent to the disc becomes hyperintense because of the existing bone marrow edema. Furthermore, MRI has the highest sensitivity for differentiation between inactive and active cases. In asymptomatic patients with inactive disease, the affected lesion has a hypointense signal both in T1- and T2weighted MRI because of inactivity.

\section{Treatment}

Appropriate treatment is mandatory for SP in patients with intractable back pain and neurological deficits, but asymptomatic cases only require periodic evaluation. Treatment for symptomatic pseudarthrosis is either conservative, with immobilization in braces, or surgical, but the strategy should be individualized., 72,56

Conservative Treatment. In conservative treatment, no trial showing the ideal duration of immobilization exists. However, this strategy works better in less mobile thoracic segments than in the more mobile thoracolumbar and cervical spine. In such cases, although amelioration of pain might occur with conservative treatment, healing and bony bridging is less likely despite a high capacity for healing in ankylosing diseases.

Surgery. The principal management for symptomatic pseudarthrosis that fails to resolve with conservative treatment is surgery. In addition, surgery is highly recommended when extensive destruction in association with neurological involvement exists, in particular in mobile segments of the thoracolumbar and lumbar regions. The most effective and safe surgical procedure for symptomatic thoracolumbar pseudarthrosis is still controversial. Several methods of surgery have been advocated, including posterior, anterior, and combined anterior-posterior procedures.

A thorough review of the literature revealed that 1-stage or staged combined $360^{\circ}$ anterior-posterior surgery with debridement of all necrotic tissues from the gap and placement of a cage or an appropriately shaped autograft, followed by interlaminar laminectomy and posterior instrumentation, are the most frequent type of surgical mode in the treatment of pseudarthrosis in all ankylosing conditions. ${ }^{14,37}$ This mode of surgery is useful in preventing the progression of kyphosis and in providing some correction of preexisting kyphosis. For this purpose, after debridement of the defect, with manual pushing of vertebrae anteriorly, maximum opening of the defect and its packing with an autogenous bone graft of optimal size can be achieved, so that correction of the kyphosis can be reached. The average correction of kyphosis will be $20^{\circ}$ by this means. ${ }^{14}$

Notably, because of the associated osteoporosis, a long construct or cement augmentation might be justified. Some who prefer this strategy suggest adding a Smith-Petersen osteotomy to $360^{\circ}$ surgery whenever focal kyphosis of a greater degree coexists.

The anterior-only approach with debridement and autogenous bone graft described by Fang et al. had the disadvantage of nonunion, which occurred in 2 of their 13 reported cases. ${ }^{22}$ Furthermore, canal compromise by posteriorly located fibrous tissue is not targeted.

If pseudarthrosis is associated with marked kyphotic deformity, besides achievement of solid fusion at the site of pseudarthrosis, another goal of surgical treatment should be to restore sagittal alignment and correction of the kyphotic deformity. For this purpose, opening and closing wedge osteotomies have been postulated. With consideration of the unique ability to bridge the ventral gap in AS, in 2006 Chang et al. proposed opening wedge osteotomy combined with posterior instrumentation and fusion as a sufficient mode for management of pseudarthrosis. ${ }^{13}$ They have proved that the fractured part of the vertebral body or the gap fills and ossifies with time. However, opponents of this treatment believe that surgical decompression (which is the mainstay of treatment in the presence of neurological deficit) is not targeted, although kyphosis of marked degree is corrected.

In 2006, Van Royen et al. described their method as consisting of posterior closing wedge osteotomy at the vertebra, 1 level below the site of pseudarthrosis, and cleaning of the adjacent affected disc space via the same approach. ${ }^{6}$ They believed that with this procedure both the kyphosis and the pseudarthrosis could be targeted, resulting in correction of the former and healing of the latter. Accordingly, anterior solid fusion can occur without grafts, considering the superior healing ability that exists in all ankylosing diseases. In 2012, Qian et al. introduced closing wedge osteotomy or pedicle subtraction osteotomy at the level of the pseudarthrosis, supplemented with anterior fusion in a second stage.

The posterior-only approach, which was described in the current case, might be an acceptable alternative to previous methods, with the advantages of decreased time of surgery, blood loss, hospital stay, and obviation of the need for anterior surgery. This mode is likely preferable for those with moderate kyphosis. In a very careful review of the literature, and to the best of our knowledge, the application of this approach has not been mentioned for pseudarthrosis. This $360^{\circ}$ surgery via a posterior-only corridor can provide adequate exposure for complete debridement and, with optimal distraction, can prepare a suitable bed for placement of an appropriately shaped autogenous graft or cage. Furthermore, posterior cord decompression, establishment of anterior column height, and correction of local kyphosis become possible, and adding long-segment posterior instrumentation will guarantee the alignment and stabilization.

\section{Outcome}

Significant pain relief and improvement of clinical visual analog scale scores are usually obtained postopera- 
tively, regardless of the type of surgery. An average improvement of 4-8 points in the pain scores has usually been reported. Furthermore, the majority of patients with neurological deficits usually show marked improvement after the operation. Up to $87.5 \%$ of patients are quite satisfied and can return to their previous activities in less than 3 months after surgery.

\section{Conclusions}

With the appearance of sharp and severe local back pain, SP should be considered in all patients who have already suffered from an ankylosing disorder. It should be noted that this complication is not confined to patients with AS, DISH, psoriasis, and IBD, but can affect other states such as ochronosis-although this is rare. Furthermore, the imaging investigation of a patient with inactive stiff-backed disease is necessary once local back pain of severe intensity appears. Unbearable pain and progressive neurological deficits are the indications for surgery. With increased popularity of the posterior-only approach, which has recently been applied in different spinal pathologies, the proposed corridor might be an alternative option in the management of pseudarthrosis, instead of combined anterior-posterior surgery.

\section{References}

1. Abe Y, Oshima N, Hatanaka R, Amako T, Hirohata R: Thirteen cases of alkaptonuria from one family tree with special reference to osteo-arthrosis alkaptonurica. J Bone Joint Surg Am 42-A:817-831, 1960

2. Agarwal AK, Reidbord HE, Kraus DR, Eisenbeis CH Jr: Variable histopathology of discovertebral lesion (spondylodiscitis) of ankylosing spondylitis. Clin Exp Rheumatol 8:67-69, 1990

3. Akeda K, Kasai Y, Kawakita E, Matsumura Y, Kono T, Murata T, et al: Thoracic myelopathy with alkaptonuria. Spine (Phila Pa 1976) 33:E62-E65, 2008

4. Al-Mahfoudh R, Clark S, Buxton N: Alkaptonuria presenting with ochronotic spondyloarthropathy. Br J Neurosurg 22:805-807, 2008

5. Balaban B, Taskaynatan M, Yasar E, Tan K, Kalyon T: Ochronotic spondyloarthropathy: spinal involvement resembling ankylosing spondylitis. Clin Rheumatol 25:598-601, 2006

6. Bayindir P, Yilmaz Ovali G, Pabuşçu Y, Temiz C, Duruoz T: Radiologic features of lumbar spine in ochronosis in late stages. Clin Rheumatol 25:588-590, 2006

7. Bron JL, de Vries MK, Snieders MN, van der Horst-Bruinsma IE, van Royen BJ: Discovertebral (Andersson) lesions of the spine in ankylosing spondylitis revisited. Clin Rheumatol 28:883-892, 2009

8. Calin A, Robertson D: Spondylodiscitis and pseudarthrosis in a patient with enteropathic spondyloarthropathy. Ann Rheum Dis 50:117-119, 1991

9. Canhão H, Coelho P, Pereira da Silva J, Santos MJ, Teixeira da Costa J, Viana de Queiroz M: Does coexistence of ochronosis and ankylosing spondylitis really exist? Clin Exp Rheumatol 14:699-703, 1996

10. Capkin E, Karkucak M, Yayli S, Serdaroğlu M, Tosun M: Ochronosis in differential diagnosis of patients with chronic backache: a review of the literature. Rheumatol Int 28:6164, 2007

11. Cawley MI, Chalmers TM, Kellgren JH, Ball J: Destructive lesions of vertebral bodies in ankylosing spondylitis. Ann Rheum Dis 31:345-358, 1972
12. Chan FL, Ho EK, Chau EM: Spinal pseudarthrosis complicating ankylosing spondylitis: comparison of CT and conventional tomography. AJR Am J Roentgenol 150:611-614, 1988

13. Chang KW, Tu MY, Huang HH, Chen HC, Chen YY, Lin CC: Posterior correction and fixation for pseudoarthrosis with kyphotic deformity in ankylosing spondylitis. Spine (Phila Pa 1976) 31:E408-E413, 2006

14. Chen LH, Kao FC, Niu CC, Lai PL, Fu TS, Chen WJ: Surgical treatment of spinal pseudoarthrosis in ankylosing spondylitis. Chang Gung Med J 28:621-628, 2005

15. Choudhury R, Rajamani SS, Rajshekhar V: A case of ochronosis: MRI of the lumbar spine. Neuroradiology 42:905907,2000

16. De Keyser F, De Paepe A, Veys EM: Ochronotic arthropathy and ankylosing spondylitis in an HLA-B27 positive patient. J Orthop Rheumatol 3:119-120, 1990 (Letter)

17. Deeb Z, Frayha RA: Multiple vacuum discs, and early sign of ochronosis. Radiologic findings in two brothers. J Rheumatol 3:82-87, 1976

18. Dihlmann W, Delling G: Disco-vertebral destructive lesions (so-called Andersson lesions) associated with ankylosing spondylitis. Skeletal Radiol 3:10-16, 1978

19. Effelsberg NM, Hügle T, Walker UA: A metabolic cause of spinal deformity. Metabolism 59:140-143, 2010

20. Emel E, Karagöz F, Aydín IH, Hacísalihoğlu S, Seyithanoğlu MH: Alkaptonuria with lumbar disc herniation: a report of two cases. Spine (Phila Pa 1976) 25:2141-2144, 2000

21. Eschelman DJ, Beers GJ, Naimark A, Yablon I: Pseudoarthrosis in ankylosing spondylitis mimicking infectious diskitis: MR appearance. AJNR Am J Neuroradiol 12:11131114, 1991

22. Fang D, Leong JCY, Ho EK, Chan FL, Chow SP: Spinal pseudoarthrosis in ankylosing spondylitis. Clinicopathological correlation and the results of anterior spinal fusion. $\mathbf{J}$ Bone Joint Surg Br 70:443-447, 1988

23. Farzannia A, Shokouhi G, Hadidchi S: Alkaptonuria and lumbar disc herniation. Report of three cases. J Neurosurg 98 (1 Suppl):87-89, 2003

24. Feild JR, Higley GB Sr, Desaussure RL Jr: Ochronosis with ruptured lumbar disc. Case report. J Neurosurg 20:348-351, 1963

25. Gaines JJ Jr: The pathology of alkaptonuric ochronosis. Hum Pathol 20:40-46, 1989

26. Gemignani G, Olivieri I, Semeria R, Giustarini S, Pasero G: Coexistence of ochronosis and ankylosing spondylitis. J Rheumatol 17:1707-1709, 1990

27. Goto S, Tanno T, Moriya H: Cervical myelopathy caused by pseudoarthrosis between the atlas and axis associated with diffuse idiopathic skeletal hyperostosis. Spine (Phila Pa 1976) 20:2572-2575, 1995

28. Gürkanlar D, Daneyemez M, Solmaz I, Temiz C: Ochronosis and lumbar disc herniation. Acta Neurochir (Wien) 148:891-894, 2006

29. Hamdulay SS, Finegold J, Boyer L, Khanna M, Akmal M, Walker A, et al: Clinical images: Magnetic resonance imaging appearance of alkaptonuria. Arthritis Rheum 64:129, 2012

30. Hasegawa K, Takahashi H, Idia Y, Yokoyama Y, Fukutake K, Takamatsu R, et al: Spontaneous symptomatic pseudoarthrosis at L2-L3 with diffuse idiopathic hyperostosis. Case Rep Orthop 2013:497458, 2013

31. Jebaraj I, Chacko BR, Chiramel GK, Matthai T, Parameswaran A: A simplified staging system based on the radiological findings in different stages of ochronotic spondyloarthropathy. Indian J Radiol Imaging 23:101-105, 2013

32. Justesen P, Anderson PE Jr: Radiologic manifestations in alcaptonuria. Skeletal Radiol 11:204-208, 1984

33. Kabasakal Y, Garrett SL, Calin A: The epidemiology of spondylodiscitis in ankylosing spondylitis-a controlled study. Br J Rheumatol 35:660-663, 1996 
34. Kabasakal Y, Kiyici I, Ozmen D, Yagci A, Gumusdis G: Spinal abnormalities similar to ankylosing spondylitis in a 58-year-old woman with ochronosis. Clin Rheumatol 14:355-357, 1995

35. Kalevski SK, Haritonov DG, Peev NA: Alcaptonuria with lumbar disc prolapse: case study and review of the literature. Spine J 7:495-498, 2007

36. Kenny JB, Hughes PL, Whitehouse GH: Discovertebral destruction in ankylosing spondylitis: the role of computed tomography and magnetic resonance imaging. Br J Radiol 63:448-455, 1990

37. Kim KT, Lee SH, Suk KS, Lee JH, Im YJ: Spinal pseudoarthrosis in advanced ankylosing spondylitis with sagittal plane deformity: clinical characteristics and outcome analysis. Spine (Phila Pa 1976) 32:1641-1647, 2007

38. Kurugoglu S, Mihmanli I, Kanberoglu K, Kanberoglu A: Destructive diskovertebral lesions in ankylosing spondylitis: appearance on magnetic resonance imaging. South Med J 94:837-841, 2001

39. Lagier R, Sit'aj S: Vertebral changes in ochronosis. Ann Rheum Dis 33:86-92, 1974

40. Laskar FH, Sargison KD: Ochronotic arthropathy. A review with four case reports. J Bone Joint Surg Br 52:653-666, 1970

41. Li KJ, Yu CL, Hsu CY, Hsieh SC, Hsu PN: Spinal pseudoarthrosis: a rare complication in psoriatic arthritis. J Formos Med Assoc 105:685-688, 2006

42. Little H, Urowitz MB, Smythe HA, Rosen PS: Asymptomatic spondylodiscitis. An unusual feature of ankylosing spondylitis. Arthritis Rheum 17:487-493, 1974

43. Mannoni A, Selvi E, Lorenzini S, Giorgi M, Airó P, Cammelli D, et al: Alkaptonuria, ochronosis, and ochronotic arthropathy. Semin Arthritis Rheum 33:239-248, 2004

44. McCollum DE, Odom GL: Alkaptonuria, ochronosis, and low-back pain. A case report. J Bone Joint Surg Am 47:1389-1392, 1965

45. Miyamoto K, Shimizu K, Arimoto R, Sakaguchi Y, Nishimoto H, Kodama H, et al: Spontaneous symptomatic pseudoarthrosis at the T11-T12 intervertebral space with diffuse idiopathic skeletal hyperostosis: a case report. Spine (Phila Pa 1976) 28:E320-E322, 2003

46. O'Brien WM, Banfield WG, Sokoloff L: Studies on the pathogenesis of ochronotic arthropathy. Arthritis Rheum 4:137-152, 1961

47. Onda A, Kikuchi S, Yabuki S, Otani K, Konno S: A case of thoracic myelopathy secondary to alkaptonuric spondylosis. J Orthop Sci 17:495-499, 2012

48. Palazzi C, D’Angelo S, Leccese P, Nigro A, Olivieri I: Ochronotic arthropathy of the spine limited to the thoracic section. Rheumatology (Oxford) 52:799, 2013

49. Park WM, Spencer DG, McCall IW, Ward J, Buchanan WW, Stephens WH: The detection of spinal pseudarthrosis in ankylosing spondylitis. Br J Radiol 54:467-472, 1981

50. Pastershank SP, Resnick D: Pseudoarthrosis in ankylosing spondylitis. J Can Assoc Radiol 31:234-235, 1980

51. Pegan-Carlo J, Payzant AR: Roentgenographic manifestations in a severe case of alkaptonuric osteoarthrosis. AJR Am J Roentgenol 80:519-521, 1960

52. Peh WCG, Ho TK, Chan FL: Pseudoarthrosis complicating ankylosing spondylitis - appearances on magnetic resonance imaging. Clin Radiol 47:359-361, 1993

53. Peh WCG, Luk KD: Pseudoarthrosis in ankylosing spondylitis. Ann Rheum Dis 53:206-210, 1994

54. Peretz A, Dupont M, Famaey JP: Ochronosis: a case report with severe ochronotic arthropathy. Clin Rheumatol 2:293 298, 1983

55. Perić $\mathrm{P}$, Curković B, Potocki K, Babić-Naglić D, Perić S, Cerovski B, et al: Coexistence of ochronosis and B 27 positive ankylosing spondylitis. Coll Antropol 31:637-640, 2007

56. Pettersson T, Laasonen L, Leirisalo-Repo M, Tervahartiala
P: Spinal pseudoarthrosis complicating ankylosing spondylitis: a report of two patients. Br J Rheumatol 35:1319-1323, 1996

57. Phornphutkul C, Introne WJ, Perry MB, Bernardini I, Murphey MD, Fitzpatrick DL, et al: Natural history of alkaptonuria. N Engl J Med 347:2111-2121, 2002

58. Pomeranza MM, Friedman LJ, Tunick IJ: Roentgen findings in alkaptonuric ochronosis. Radiology 37:295-302, 1941

59. Pratibha K, Seenappa T, Ranganath K: Alkaptonuric ochronosis: Report of a case and brief review. Indian J Clin Biochem 22:158-161, 2007

60. Qian BP, Qiu Y, Wang B, Sun X, Zhu ZZ, Jiang J, et al: Pedicle subtraction osteotomy through pseudarthrosis to correct thoracolumbar kyphotic deformity in advanced ankylosing spondylitis. Eur Spine J 21:711-718, 2012

61. Quagliano PV, Hayes CW, Palmer WE: Vertebral pseudoarthrosis associated with diffuse idiopathic skeletal hyperostosis. Skeletal Radiol 23:353-355, 1994

62. Reddy DR, Prasad VS: Alkaptonuria presenting as lumbar disc prolapse: case report and review of literature. Spinal Cord 36:523-524, 1998

63. Sag AA, Silbergleit R, Olson RE, Wilson J, Krishnan A: T1 hyperintense disc in alkaptonuria. Spine (Phila Pa 1976) 37:E1361-E1363, 2012

64. Schilling F, Stollenwerk R, Dreher R: Traditional and new types of spondarthritis with special consideration of spondylodiscitis. Neurosurg Rev 13:273-278, 1990

65. Schweitzer ME, el-Noueam KI: Vacuum disc: frequency of high signal intensity on T2-weighted MR images. Skeletal Radiol 27:83-86, 1998

66. Shen FH, Samartzis D: Successful nonoperative treatment of a three-column thoracic fracture in a patient with ankylosing spondylitis: existence and clinical significance of the fourth column of the spine. Spine (Phila Pa 1976) 32:E423-E427, 2007

67. Shih TT, Chen PQ, Li YW, Hsu CY: Spinal fractures and pseudoarthrosis complicating ankylosing spondylitis: MRI manifestation and clinical significance. J Comput Assist Tomogr 25:164-170, 2001

68. Unsal E, Arici AM, Kavukçu S, Pirnar T: Andersson lesion: spondylitis erosiva in adolescents. Two cases and review of the literature. Pediatr Radiol 32:183-187, 2002

69. Van Royen BJ, Kastelijns RC, Noske DP, Oner FC, Smit TH: Transpedicular wedge resection osteotomy for the treatment of a kyphotic Andersson lesion-complicating ankylosing spondylitis. Eur Spine J 15:246-252, 2006

70. Watts RWE, Watts RA: Alkaptonuria: a 60-yr follow-up. Rheumatology (Oxford) 46:358-359, 2007

71. Wu PC, Fang D, Ho EK, Leong JC: The pathogenesis of extensive discovertebral destruction in ankylosing spondylitis. Clin Orthop Relat Res (230):154-161, 1988

\section{Disclosures}

The authors report no conflict of interest concerning the materials or methods used in this study or the findings specified in this paper.

\section{Author Contributions}

Conception and design: Abolfazl Rahimizadeh. Acquisition of data: Abolfazl Rahimizadeh, Ava Rahimizadeh. Drafting the article: Soufiani, Hassani, Ava Rahimizadeh. Critically revising the article: Hassani.

\section{Correspondence}

Abolfazl Rahimizadeh, Department of Neurosurgery, PAMIM Research Center, Pars Hospital, 83 Keshawarz Blvd., Tehran 15145, Iran. email: a_rahimizadeh@hotmail.com. 